\title{
Temporal Aspects of Global Coherence during Discourse Production in Early Stage Parkinson's Disease
}

\author{
Charles Ellis ${ }^{1}$, Xiangming Fang' ${ }^{2}$ Patrick Briley ${ }^{1}$ \\ ${ }^{1}$ Department of Communication Sciences and Disorders, East Carolina University, Greenville, NC, USA \\ ${ }^{2}$ Department of Biostatistics, East Carolina University, Greenville, NC, USA \\ Email: ellisc14@ecu.edu, fangx@ecu.edu, brileypa@ecu.edu
}

Received 22 June 2016; accepted 23 July 2016; published 26 July 2016

Copyright (C) 2016 by authors and Scientific Research Publishing Inc.

This work is licensed under the Creative Commons Attribution International License (CC BY). http://creativecommons.org/licenses/by/4.0/

(c) (†) Open Access

\begin{abstract}
Introduction: Parkinson's Disease (PD) is a devastating neurodegenerative disease that significantly influences motor performance. Recent studies suggest expressive language deficits may also exist among individuals with PD even though the condition is primarily known for changes in motor skills. The purpose of this pilot study was to examine the influence of early stage PD on global coherence or the ability to maintain consistency of a theme or topic. Methods: Discourse samples were collected from eleven individuals with PD and compared to eleven matched controls to examine measures of global coherence. Two-minute speech samples describing a "typical day" were transcribed and rated on mean global coherence and percentage high global coherence. Results: Greater declines in global coherence were observed among individuals with PD, however, comparisons of both mean global coherence ratings and percentage high global coherence were not statistically significant between the two groups. A significant downward linear trend was observed for both mean global coherence ratings and percentage high coherence among all participants indicating decreases in global coherence over time. Conclusion: Approaches to the study of global coherence as a measure of expressive language performance should consider the temporal changes especially among neurological conditions known to reduce expressive language ability.
\end{abstract}

\section{Keywords}

Parkinson's Disease, Basal Ganglia, Discourse, Language, Global Coherence

\section{Introduction}

Parkinson's Disease (PD) is a neurodegenerative syndrome that has a world-wide crude incidence rate of 4.5 -

How to cite this paper: Ellis, C., Fang, X.M. and Briley, P. (2016) Temporal Aspects of Global Coherence during Discourse Production in Early Stage Parkinson's Disease. Advances in Parkinson's Disease, 5, 41-49. 
19 per 100,000 [1]. The economic cost of PD is substantial and poses a substantial burden to individuals with PD, their families and society [2]. According to the National Parkinson Foundation, 50,000 - 60,000 new cases are diagnosed in the United States annually [3].

The most obvious signs of PD are related to reductions in motor performance resulting from a disease process that occurs in the basal ganglia. However, many individuals with PD experience non-motor signs such as nocturia, fatigue and forgetfulness that contribute to decreases in overall quality of life [4]. The complexity of the disease process is due to a disease progression that courses through the basal ganglia (BG), brainstem and the cerebral cortex [5]. Many individuals with PD also experience non-motor cognitive deficits because of the extensive and progressive nature of the disease process [6]-[8]. Traditionally, cognition has received less attention in the literature in part because motor issues are much more noticeable in the early disease stages than non-motor disease symptoms.

\subsection{Neuropathology of PD}

The neuropathological process that occurs in PD suggests that non-motor symptoms should co-occur with the more visible motor deficits. The primary PD disease process centers on the BG, a collection of nuclei that are connected to the cerebral cortex via a collection of cortical-BG-thalamic-cortical circuits that vary in function [9] [10]. The circuitry of the BG serves as the anatomical bases for potential non-motor deficits in cognition and also language both traditionally believed to be governed by the cerebral cortex [11] [12]. The extensive connections between the BG and cerebral cortex serve as the basis for the occurrence of hypothesized expressive language deficits in PD. According to BG models, PD disrupts cortical-BG-thalamic-cortical circuits believed critical to expressive language performance. As evidence, studies have shown that expressive language disruptions can occur in PD in the form of morphosyntactic, lexical semantic and language production breakdowns [13] [14].

\subsection{Expressive Language in PD}

Expressive language issues in PD are believed to develop concurrently with motor issues as part of the neuropathological process that disrupts the cortical-BG-thalamic-cortical circuits [5] [9] [10] [15] [16]. However, language deficits have not been observed at the same frequency as motor deficits. It is possible that non-motor expressive language symptoms are: 1) not disrupted; 2) not severe enough to be observed during the early disease stages when motor symptoms emerge; or 3) traditional measures are not diagnostically sensitive to the expected expressive language deficits at the earliest disease stages [15] [16].

\subsection{Discourse Production}

Analyses of discourse production have emerged as an approach to examine expressive language performance among individuals with neurological disease. Discourse production is a complex goal directed activity involving executive skills (intent, planning and task persistence) that involve the serial assembling of complex utterances that are determined by context and goal [11] [17]. Analyses of discourse offer researchers an opportunity to evaluate cognitive linguistic behaviors as they unfold during a natural form of communication [18]. Ash et al. (2006) noted that discourse analyses also allowed an examination of language disruptions that were independent of co-existing motor speech deficits in neurologically diseased populations [17].

\subsection{Discourse Coherence}

One specific aspect of discourse production known to be affected in neurological disease is coherence. Coherence is defined as a speaker's ability to maintain unity related to a specific theme [19] and represents the speaker's conceptual organization during discourse production [20]. Coherence can be evaluated in two ways: global coherence or the ability to organize discourse around a central theme or topic and local coherence which represents meaningful linkage between the individual sentences produced [19]. According to Coelho \& Flewellyn (2003), global coherence is believed to be a skill reflecting the overall goal, plan or topic of the discourse [20]. In contrast, local coherence is a skill that reflects the conceptual linkage between adjacent sentences designed to maintain meaning in the discourse. Coherence has been evaluated in a range of individuals with neurological disorders including: traumatic brain injury [20]-[22], stroke [17] [19] [23], Alzheimers disease [19] 
[24], frontotemporal dementia [17], Parkinson's Disease [25] and adults absent of neurological conditions [24] [26] [27].

As a first step in further understanding the nature and impact of PD on expressive language, we examined the discourse of a convenience sample of individuals with PD in the early stages of the disease. A previous study of discourse among individuals with PD found that that discourse production was more efficient and coherent when on medication vs. off medication [25]. The study highlighted that the cognitive resources supporting expressive language could be impacted by the disease even in the earliest disease stage. In particular, improvements were observed in global coherence suggesting the medications enhanced the individual's ability to maintain the topic of interest. Despite these important findings, little is known about the underlying mechanisms or specific cognitive processes that contribute to changes in discourse production after PD. Studies examining discourse production have traditionally compared one specific characteristic, at one point in time and compared to a follow-up point. The temporal aspects of global coherence offers an opportunity to evaluate whether degradation occurs over time at a rate that is greater than typically would be expected in individuals absent of disease or injury [28].

The purpose of this pilot study was to use discourse in individuals with PD and non-neurologically affected age matched controls to examine the influence of early stage PD on global coherence. The following research questions were addressed in this study:

1) Do individuals with early stage PD exhibit lower global coherence during discourse production when compared to non-neurologically impaired controls?

2) Does global coherence decline to a greater degree over time during discourse production among individuals with early stage PD?

We sought to test the hypothesis that individuals in the earliest stages of the disease would exhibit reduced global coherence and over time when compared to age and gender matched controls. Our hypothesis was based on the notion that in the earliest disease stage, individuals with PD would exhibit deficits that were primarily influenced by the BG, whereas cortical involvement occurred during the later disease stages [5]. In addition, the participants could not exhibit signs of dementia which is known to negatively influence expressive language skills. We included individuals at Hoehn \& Yahr (H \& Y) stages II and III [29]. H \& Y Stages II and III are the first neuropathological stages to present with clinical symptoms of PD and dementia is frequently absent [5] [15] [16] [29]. This study was initially reviewed and approved by the University of Florida Institutional Review Board (IRB) and subsequently reviewed and approved for secondary data analyzes by the East Carolina University IRB.

\section{Methods}

\subsection{Participants}

Participants were eleven individuals diagnosed with idiopathic PD, by a movement disorders neurologist using the strict criteria of the UK Brain Bank [30], and eleven neurologically intact individuals who were age, education, ethnicity and gender matched (hereafter referred to as control subjects). Participants were recruited from the North Florida South Ga Veterans Administration Medical Center Movement Disorders Clinic. PD participants exhibited a minimum of 3 of 4 cardinal features of PD (resting tremor, rigidity, bradykinesia, postural instability) and had no history of deep brain stimulation or brain lesion therapy. All participants (experimental and control) had at least a seventh grade education, functional hearing for normal conversation, functional vision for reading tasks, used English as their primary language, and demonstrated expressive language skills within intact range for normal conversation. All participants also exhibited scores of 26 or better on the Mini Mental Status Exam (MMSE) [31].

\subsection{Baseline Assessments}

Baseline assessments were completed by the primary author in the patient's homes. The Boston Naming Test (BNT) [32] and Wechsler Memory Scale-Logical Memory I (WMS-LMI) [33] were administered to examine potential group differences relative to naming (BNT) and the influence of short term memory on language production (WMS-LMI). 


\subsection{Collection of Discourse Samples}

Discourse samples were collected by the primary author from PD participants in their homes prior to consumption of their first daily dose of anti-parkinsonian medication and at least 12 hours after their last dose. Collecting samples prior to their first daily dose of anti-parkinsonian medication ensured they were in their "off" medication state to maximize dopamine depletion, a major putative cause of cognitive dysfunction in PD. Control participants were also examined in their homes. All participants were instructed to discuss a "typical day", and participants were asked to speak for a minimum of three minutes. In the event that subjects stopped before 3-minutes, a standardized verbal cue ("Tell me more about that") was provided to prompt a continuation of the narrative until the 3-minute minimum was achieved. A Sony VN-480 PC digital voice recorder was used to record each subject's samples.

\subsection{Transcription and Segmentation}

The first three minutes of all language samples were transcribed verbatim. Each sample was divided into communication units (CU); defined as the shortest allowable independent clause and related dependent clauses. Individual CU's were defined primarily by syntax, however prosodic and semantic features were used at times when the unit could not be determined entirely by syntax. All unintelligible words were excluded from the analysis. In instances where the location of coordinating conjunctions such as "and", "but" and "or" was unclear, their prosodic feature determined their final location at the beginning or ending of the communication unit. Oneword responses were not considered in the communication unit calculation. Scoring guidelines for communication units were based on Hunt's procedure [34].

\subsection{Global Coherence Evaluation}

Only the first two minutes of the samples were used for group comparisons of global coherence to ensure that samples of similar characteristics (word and sentence productivity) were analyzed across participants. A graduate student trained to complete global coherence ratings completed the global coherence ratings of the communication units in all samples. Each communication unit within the 2-minute samples were rated using a fivepoint global coherence scale as originally described by Glosser and Deser [19], and later adapted by Van Leer \& Turkstra [22]. Raters were instructed to use only scores of 1 (no relationship), 3 (possible relationship) and 5 (definite relationship). This method was chosen for the current study because in previous work by Van Leer \& Turkstra [19] and the first author have found that the scores two and four were rarely used. The numerical rating of global coherence for each communication unit was written on the transcription form.

Global coherence measures were calculated using two approaches. First, the mean global coherence rating was calculated for the 2-minute sample. Second, the percentage of communication units receiving a numerical rate of five or "high global coherence" was calculated for the 2-minute. To further evaluate the temporal aspects of global coherence, we calculated the mean global coherence ratings and the percentage of high coherence for each one minute interval for comparisons across time.

\subsection{Reliability}

The first author independently completed ratings on 3 discourse samples (14\%) of the total sample to assess inter rater reliability with the graduate student ratings. Point to point comparison as a measure of percentage agreement was completed. Inter-rater reliability was $90.4 \%$ for global coherence.

\subsection{Statistical Analysis}

SPSS 22 was used to analyze the data. Baseline comparisons of demographic, cognitive and language characteristics were completed. Group comparisons of global coherence ratings were completed using mean coherence ratings for each communication unit and the percentage of communication units that received a high global coherence rating (score of 5) for the first two minutes of the collected samples. Independent samples t-tests were completed to compare mean global coherence ratings and percentage of high coherence between the two groups. To examine the temporal aspects of global coherence, a repeated measures analysis of variance (ANOVA) was used to compare mean global coherence ratings and percentage high coherence changes from baseline, minute 
one and minute two. The criterion for significance was set at $\mathrm{p}<0.05$ for all statistical tests.

\section{Results}

Table 1 lists demographic, cognitive and language comparisons for subjects in the study. Non-significant differences were observed between the two groups for age, education, short term memory (WMS-LMI), and language form (BNT) and general cognitive ability (MMSE).

\section{Mean Global Coherence and Percentage High Global Coherence}

Comparisons of mean global coherence ratings did not yield statistically significant differences between the two groups. The controls exhibited higher but non-significant differences in mean global coherence ratings when compared to individuals with PD (4.03 vs. 3.58; $\mathrm{p}=0.19)$. Similarly, controls exhibited a higher percentage of communication units that received a high global coherence rating (score of 5) when compared to individuals with PD (74\% vs. 58\%; $\mathrm{p}=0.08)$.

Both groups exhibited a statistically significant downward linear trend for both mean global coherence ratings and percentage high global coherence, indicating decreases in global coherence overall ( $\mathrm{p}<0.001)$. However, comparisons of temporal changes of mean global coherence ratings did not yield statistically significant differences between the two groups. Controls declined from baseline to a mean of 4.25 during minute 1 and 3.78 in minute 2 compared to individuals with PD who declined to 3.80 during minute 1 and 3.49 in minute $2(\mathrm{p}=0.28)$ (see Figure 1). Similarly non-significant differences were observed in percentage high global coherence. Controls declined from baseline to $80.2 \%$ in minute 1 and $66.8 \%$ in minute 2 compared to individuals with PD who declined to $62.7 \%$ in minute 1 and $56.1 \%$ in minute $2(p=0.12$ ) (see Figure 2).

\section{Discussion}

The purpose of this pilot study was to examine the influence of early stage Parkinson's Disease on global coherence during discourse production. We hypothesized that individuals with early stage PD, absent of dementia, would exhibit lower global coherence than age and gender matched non-neurologically matched controls even in the earliest stage of PD. Our hypothesis was based on the notion that the disease process for PD has been shown to influence expressive language performance which is governed by a complex interaction of cortical and subcortical structures. For this project, we calculated both mean rating scores and average percent high global coherence.

Two key findings emerged from this project. The first key finding was that global coherence does not appear to be influenced by PD in the earliest disease stages to a greater degree than non-neurologically matched controls. Although deficits in global coherence have been observed in other neurologically impaired populations

Table 1. Demographic, cognitive, and language comparisons for PD and control subjects.

\begin{tabular}{cccccc} 
& \multicolumn{2}{c}{$\begin{array}{c}\text { PD subjects } \\
(\mathrm{N}=11)\end{array}$} & \multicolumn{2}{c}{$\begin{array}{c}\text { Controls } \\
(\mathrm{N}=11)\end{array}$} & $\mathrm{p}$ \\
\cline { 2 - 5 } Variable & $\mathrm{M}$ & $\mathrm{SD}$ & $\mathrm{M}$ & $\mathrm{SD}$ & \\
Age & 70.6 & 13.1 & 71.2 & 13.2 & 0.92 \\
Education & 12.0 & 1.3 & 12.9 & 2.9 & 0.36 \\
Parkinson Years & 4.0 & 4.7 & & & \\
H \& Y stage & 2.5 & 0.5 & & & \\
BNT & 52.7 & 7.2 & 53.2 & 7.1 & 0.88 \\
MMSE & 28.8 & 1.3 & 29.0 & 1.5 & 0.77 \\
WMS-LMI & 29.3 & 11.3 & 32.0 & 14.2 & 0.62 \\
\hline
\end{tabular}

Parkinson Years = the number of years since PD subjects were initially diagnosed; H \& Y = Hoehn and Yahr; BNT = Boston Naming Test, all items administered; MMSE = Mini Mental Status Exam; WMS-LMI = Wechsler Memory Scale-Logical Memory I subtest. 


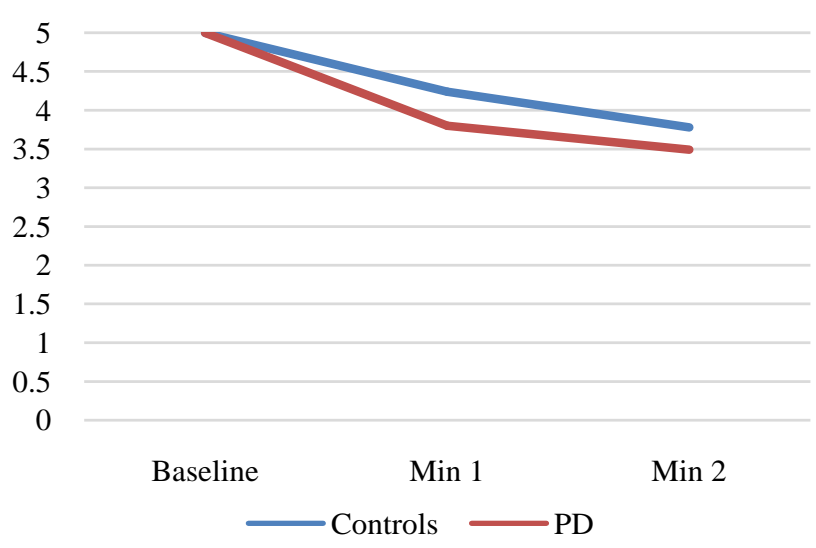

Figure 1. Group comparisons of temporal changes in mean global coherence.

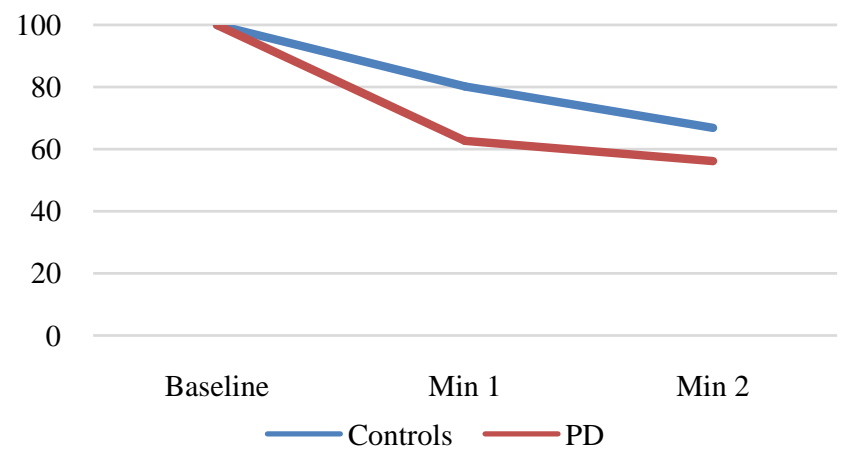

Figure 2. Group comparisons of temporal changes in percent high global coherence.

(traumatic brain injury, stroke, Alzheimers disease and frontotemporal dementia) and in normal aging, the findings here do not support significant disruptions of global coherence in early stage PD. It is possible that global coherence is primarily governed by cortical involvement and individuals with diseases such as PD, primarily limited to subcortical structures in the earliest stages of the disease, do not disrupt global coherence. This position is supported by research that has shown the disease process begins subcortically, courses through the BG and other subcortical structures, and then affects the brainstem and the cerebral cortex in the later disease stages [5]. These findings further highlight the multifactorial nature of deficits in PD and the need for additional studies at different disease stages to explain the nature of expressive language performance during this disease process [35].

The second key finding was global coherence declines in a temporal fashion during discourse production. Both groups (PD and controls) exhibited declines in global coherence regardless of whether the score was calculated by mean rating or percent high coherence. These observations were seen in both groups as their discourse progressed from minute 1 to minute 2 (see Figure 1 and Figure 2). It is unclear if this phenomenon is to be typically expected as previous studies have not considered these temporal changes. It should be noted that the two minute samples included here were longer than typically observed during studies of global coherence. Examinations of global coherence have been traditionally limited to picture descriptions and short story retelling, thereby limiting the discourse output. Therefore, it is possible that such tasks are potentially overestimating global coherence in disease populations particularly in relationship to real world communication [36]. Future studies are needed due the importance of global coherence during discourse production particularly for social interactions and general communication [17].

To our knowledge, no previous studies have examined the temporal aspects of global coherence in PD during the earliest disease stages. This study offers information regarding the need for future investigations to better understand global coherence in discourse and the influence of progressive diseases such as PD. Traditional measures of global coherence have been completed using scales to rate the individual communication units, whereby an average mean score or percent high global coherence score is calculated. Studies are needed to fur- 
ther explain the temporal aspects of global coherence in normal and neurologically impaired populations. Similarly, studies are needed to examine global coherence in discourse using a variety of discourse tasks (independent story generation of past history, retelling of stories, procedural tasks such as cooking a meal) and samples varying in lengths [36]. Examining global coherence in a range of populations and using a variety of discourse types will offer additional information about the nature of global coherence and the mechanisms that negatively impact the maintenance of this skill.

This study highlights the need to further explore approaches to the measurement of global coherence. Measures of mean global coherence and percentage of "high" global coherence have been frequent in the literature, however, they may represent two distinct outcomes. Measuring global coherence using mean ratings across utterances represents the speakers overall average performance. The average overall performance may not offer a clear indication of the speaker's performance as higher rating scores at critical junctions of discourse could raise the overall performance score. Yet at the same time, the discourse produced may not be representative of successful output that is highly globally coherent. Similarly, measures of percent of high global coherence which represent the percentage of communication units that were rated as "highly globally coherence" or the presentation of information that is "substantially related to the general topic of discussion" are only measures of the percentage of high coherent information produced. Similar to mean ratings, they may not take into account whether information communicated at specific time points or junctures during the discourse production occurred in a necessary time or format to be perceived by the listener as "substantive information to the general topic of discussion”.

This study has a number of limitations. First, this is a pilot study with a small sample size, thereby limiting the generalization to the larger PD population. Additional details may be obtained from a larger sample of individuals with PD and a collection of discourse samples of longer length. The findings reported here should be interpreted with caution given the size of the sample reported. Second, samples were collected only during the "off" medication state. Additional insights may have been learned by also collecting data during the "on" medication state. Sanchez and Spencer (2013) found individuals with PD produced more efficient and coherent discourse when "on" medication versus "off" medication [25]. Third, although PD participants were all at H \& Y stages 2 - 3, the average disease duration for the sample was greater than 4 years. Therefore, some of the PD participants had experienced the disease for substantially longer periods than others who were newly diagnosed. Future studies should consider a more homogenous sample in terms of disease stage and disease duration. Despite these limitations, this pilot study offers foundational information for future studies of expressive language performance and specifically discourse production in PD.

\section{Conclusion}

Studies on individuals with PD have primarily focused on motor speech deficits, whereas less attention has been given to expressive language skills. Yet, changes in expressive language ability can impact the psychological and social functioning of the person with PD and their caregivers [37]. Models of PD disease suggest language may be impacted to a greater degree than previously reported. Studies are needed to not only examine the temporal aspects of expressive language performance but also to examine individuals with PD at different and progressing disease stages to determine the impact of the disease over time.

\section{Acknowledgements}

A Pre-Doctoral Fellowship from the VA Office of Academic Affairs awarded to the first author while a predoctoral fellow in the Brain Rehabilitation Research Center, VAMC, Gainesville, FL to collect the data reported in this project.

\section{References}

[1] World Health Organization (2006) Neurological Disorders: Public Health Challenges. 140-150. http://www.who.int/mental_health/neurology/neurological_disorders_report_web.pdf

[2] Mateus, C. and Coloma, J. (2013) Health Economics and Cost of Illness in Parkinson's Disease. European Neurological Reviews, 8, 6-9. http://dx.doi.org/10.17925/ENR.2013.08.01.6

[3] National Parkinson Foundation (2016). http://www.parkinson.org/sites/default/files/Parkinsonism.pdf 
[4] Stocchi, F., Martinez-Martin, P. and Reichmann, H. (2014) Quality of Life in Parkinson’s Disease-Patient, Clinical and Research Perspectives. European Neurological Reviews, 9, 12-18. http://dx.doi.org/10.17925/ENR.2014.09.01.12

[5] Braak, H., Del Tredici, K., Rub, U., de Vos, R.A., Jansen Steur, E.N. and Braak, E. (2003) Staging of Brain Pathology Related to Sporadic Parkinson's Disease. Neurobiology of Aging, 24, 197-211. http://dx.doi.org/10.1016/S0197-4580(02)00065-9

[6] Aarsland, D., Bronnick, K., Larsen, J.P., Tysnes, O.B., Alves, G., and for the Norweigian Park West Study Group (2009) Cognitive Impairment in Incident Untreated Parkinson Disease. Neurology, 72, 1121-1126. http://dx.doi.org/10.1212/01.wnl.0000338632.00552.cb

[7] Williams-Gray, C.H., Foltynie, T., Brayne, C.E.G., Robbins, T.W. and Barker, R.A. (2007) Evolution of Cognitive Dysfunction in an Incident Parkinson's Disease Cohort. Brain, 130, 1787-1798. http://dx.doi.org/10.1093/brain/awm111

[8] Litvan, I., Aarsland, D., Adler, C.H., Goldman, J.G., Kulisevsky, J., Mollenhauer, B., et al. (2011) MDS Task Force on Mild Cognitive Impairment in Parkinson’s Disease: Critical Review of PD-MCI. Movement Disorders, 26, 1814-1824. http://dx.doi.org/10.1002/mds.23823

[9] Middleton, F.A. and Strick, P.L. (2000) Basal Ganglia and Cerebellar Loops: Motor and Cognitive Circuits. Brain Research Reviews, 31, 236-250. http://dx.doi.org/10.1016/s0165-0173(99)00040-5

[10] Middleton, F.A. and Strick, P.L. (2000) Basal Ganglia Output and Cognition: Evidence from Anatomical, Behavioral, and Clinical Studies. Brain and Cognition, 42, 183-200. http://dx.doi.org/10.1006/brcg.1999.1099

[11] Alexander, M.P. (2002) Disorders of Language after Frontal Lobe Injury: Evidence for the Neural Mechanisms of Assembling Language. In: Stuss, D.T. and Knight, R.T., Eds., Principles of Frontal Lobe Function, Oxford University Press, New York, 159-167. http://dx.doi.org/10.1093/acprof:oso/9780195134971.003.0010

[12] Salmon, D.P., Heindel, W.C. and Hamilton, J.M. (2001) Cognitive Abilities Mediated by Frontal-Subcortical Circuits. In: Litcher, D.G. and Cummings, J.L., Eds., Frontal-Subcortical Circuits in Psychiatric and Neurological Disorders, Guilford Press, New York, 114-150.

[13] Murray, L. (2008) Language and Parkinson’s Disease. Annual Review of Applied Linguistics, 28, 113-127. http://dx.doi.org/10.1017/S0267190508080100

[14] Altmann, L.J. and Troche M.S. (2011) High-Level Language Production in Parkinson’s Disease: A Review. Parkinsons Disease, 2011, Article ID: 238956.

[15] Braak, H., Rub, U. and Del Tredici, K. (2006) Cognitive Decline Correlates with Neuropathological Stage in Parkinson's Disease. Journal of Neurological Sciences, 248, 255-258. http://dx.doi.org/10.1016/j.jns.2006.05.011

[16] Braak, H., Rub, U., Jansen Steur, E.N., Del Tredici, K. and de Vos, R.A. (2005) Cognitive Status Correlates with Neuropathologic Stage in Parkinson Disease. Neurology, 64, 1404-1410. http://dx.doi.org/10.1212/01.WNL.0000158422.41380.82

[17] Ash, S., Moore, P., Antani, S., McCawley, G., Work, M. and Grossman, M. (2006) Trying to Tell a Tale: Discourse Impairments in Progressive Aphasia and Frontotemporal Dementia. Neurology, 66, 1405-1413. http://dx.doi.org/10.1212/01.wnl.0000210435.72614.38

[18] Fergadiotis, G. and Wright, H.H. (2011) Lexical Diversity for Adults with and without Aphasia across Discourse Elicitation Tasks. Aphasiology, 25, 1414-1430. http://dx.doi.org/10.1080/02687038.2011.603898

[19] Glosser, G. and Deser, T. (1991) Patterns of Discourse Production among Neurological Patients with Fluent Language Disorders. Brain and Language, 40, 67-88. http://dx.doi.org/10.1016/0093-934X(91)90117-J

[20] Coelho, C.A. and Flewellyn, L. (2003) Longitudinal Assessment of Coherence in an Adult with Fluent Aphasia: A Follow-Up Study. Aphasiology, 17, 173-182. http://dx.doi.org/10.1080/729255216

[21] Hough, M.S. and Barrow, I. (2000) Descriptive Discourse Abilities of Traumatic Brain Injured Adults. Aphasiology, 17, 183-191. http://dx.doi.org/10.1080/729255221

[22] Van Leer, E. and Turkstra, L. (1999) The Effect of Elicitation Task on Discourse Coherence and Cohesion in Adolescents with Brain Injury. Journal of Communication Disorders, 32, 327-349. http://dx.doi.org/10.1016/S0021-9924(99)00008-8

[23] Rogalski, Y. and Edmonds, L.A. (2008) Attentive Reading and Constrained Summarisation (ARCS) Treatment in Primary Progressive Aphasia: A Case Study. Aphasiology, 22, 763-775. http://dx.doi.org/10.1080/02687030701803796

[24] Dijkstra, K., Bourgeois, M.S., Allen, R.S. and Burgio, L.D. (2004) Conversational Coherence: Discourse Analysis of Older Adults with and without Dementia. Journal of Neurolinguistics, 17, 263-283. http://dx.doi.org/10.1016/S0911-6044(03)00048-4

[25] Sanchez, J. and Spencer, K.A. (2013) Preliminary Evidence of Discourse Improvement with Dopaminergic Medication. Advances in Parkinson's Disease, 2, 37-42. http://dx.doi.org/10.4236/apd.2013.22007 
[26] Marini, A., Carlomagno, S., Caltagirone, C. and Nocentini, U. (2005) The Role Played by the Right Hemisphere in the Organization of Complex Textual Structures. Brain and Language, 93, 46-54. http://dx.doi.org/10.1016/j.bandl.2004.08.002

[27] Wright, H.H., Koutsoftas, A.D., Capilouto, G.J. and Fergadiotis, G. (2014) Global Coherence in Younger and Older Adults: Influence of Cognitive Processes and Discourse Type. Aging, Neuropsychology, and Cognition: A Journal on Normal and Dysfunctional Development, 21, 174-196. http://dx.doi.org/10.1080/13825585.2013.794894

[28] Ellis, C., Henderson, A., Wright, H.H. and Rogalski, Y. (2016) Global Coherence during Discourse Production in Adults: A Review of the Literature. International Journal of Language \& Communication Disorders, 51, 359-367. http://dx.doi.org/10.1111/1460-6984.12213

[29] Hoehn, M.M. and Yahr, M.D. (1967) Parkinsonism: Onset, Progression and Mortality. Neurology, 17, 427-442. http://dx.doi.org/10.1212/WNL.17.5.427

[30] Bower, J.H., Maraganore, D.M., McDonnell, S.K. and Rocca, W.A. (2000) Influence of Strict, Intermediate, and Broad Diagnostic Criteria on the Age- and Sex-Specific Incidence of Parkinson's Disease. Movement Disorders, 15, 819-825. http://dx.doi.org/10.1002/1531-8257(200009)15:5<819::AID-MDS1009>3.0.CO;2-P

[31] Folstein, M.F., Folstein, S.E. and McHugh, P.R. (1975) "Mini-Mental State”. A Practical Method for Grading the Cognitive State of Patients for the Clinician. Journal of Psychiatric Research, 12, 189-198. http://dx.doi.org/10.1016/0022-3956(75)90026-6

[32] Kaplan, E., Goodglass, H. and Weintraub, S. (1983) Boston Naming Test. Lea \& Febiger, Philadelphia.

[33] Wechsler, D. (1997) Wechsler Memory Scale. 3rd Edition Manual, Psychological Corporation, San Antonio.

[34] Hunt, K.W. (1965) Grammatical Structures Written at Three Grade Levels. National Council of Teachers of English Research Report No. 3, National Council of Teachers of English, Urbana.

[35] Batens, K., De Letter, M., Raedt, R., Duyck, W., Vanhoutte, S., Van Roost, D. and Santens, P. (2014) The Effects of Subthalamic Nucleus Stimulation on Semantic and Syntactic Performance in Spontaneous Language Production in People with Parkinson's Disease. Journal of Neurolinguistics, 32, 31-41. http://dx.doi.org/10.1016/j.jneuroling.2014.07.003

[36] Kurczek, J. and Duff, M.S. (2011) Cohesion, Coherence and Declarative Memory: Discourse Patterns in Individuals with Hippocampal Amnesia. Aphasiology, 25, 700-712. http://dx.doi.org/10.1080/02687038.2010.537345

[37] Whitehead, B. (2010) The Psychosocial Impact of Communication Changes in People with Parkinson's Disease. British Journal of Neuroscience Nursing, 6, 30-36. http://dx.doi.org/10.12968/bjnn.2010.6.1.46056

\section{Submit or recommend next manuscript to SCIRP and we will provide best service for you:}

Accepting pre-submission inquiries through Email, Facebook, LinkedIn, Twitter, etc.

A wide selection of journals (inclusive of 9 subjects, more than 200 journals)

Providing 24-hour high-quality service

User-friendly online submission system

Fair and swift peer-review system

Efficient typesetting and proofreading procedure

Display of the result of downloads and visits, as well as the number of cited articles

Maximum dissemination of your research work

Submit your manuscript at: http://papersubmission.scirp.org/ 